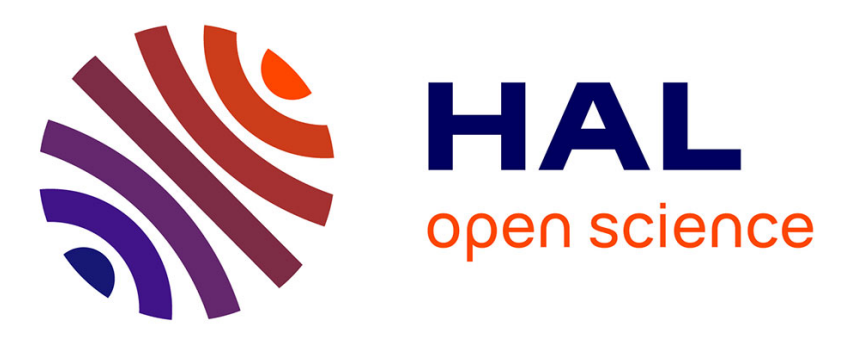

\title{
Irreversible thermodynamics and smart materials systems modelling. Example of magnetic shape memory actuators.
}

\author{
Arnaud Hubert, Nandish Calchand, Yann Le Gorrec
}

\section{To cite this version:}

Arnaud Hubert, Nandish Calchand, Yann Le Gorrec. Irreversible thermodynamics and smart materials systems modelling. Example of magnetic shape memory actuators.. IFAC Workshop on Thermodynamic foundations of Mathematical Systems Theory (TFMST'13), Jan 2013, France. pp.1-6. hal-00869587

\section{HAL Id: hal-00869587 https://hal.science/hal-00869587}

Submitted on 3 Oct 2013

HAL is a multi-disciplinary open access archive for the deposit and dissemination of scientific research documents, whether they are published or not. The documents may come from teaching and research institutions in France or abroad, or from public or private research centers.
L'archive ouverte pluridisciplinaire HAL, est destinée au dépôt et à la diffusion de documents scientifiques de niveau recherche, publiés ou non, émanant des établissements d'enseignement et de recherche français ou étrangers, des laboratoires publics ou privés. 


\title{
Irreversible Thermodynamics and Smart Materials Systems Modelling. Example of Magnetic Shape Memory actuators *
}

\author{
A. Hubert, ${ }^{*}$ N. Calchand, ${ }^{*}$ Y. Le Gorrec ${ }^{*}$ \\ * FEMTO-ST / AS2M 24, rue Alain Savary, 25000 Besançon, France \\ (e-mail: arnaud.hubert@femto-st.fr).
}

\begin{abstract}
Smart systems is a very promising technology to design high performance and highly integrated mechatronic devices. Nevertheless, one of the main drawbacks of these devices is the complex non-linear and irreversible behaviour of the active materials at the heart of such systems. To solve these difficulties, this paper presents a thermodynamics based procedure to model more accurately active materials. After a review on energy conversion modelling techniques, the paper extends classical thermodynamics procedure to the case of irreversible processes. The use of internal state variables helps to improve the physical understanding of non-linear and irreversible mechanisms in smart materials. This procedure is applied to Magnetic Shape Memory Alloys actuators and the results are quite encouraging. Modelling methods proposed in this paper improves the understanding of complex active materials for smart systems.
\end{abstract}

Keywords: Smart material systems, Actuator design and modelling, Irreversible thermodynamics, hysteretic behaviour.

\section{INTRODUCTION}

Increased demands for high performance and highly integrated mechatronic devices as well as recent advances in material science have produced a class of systems termed smart systems. The field of smart materials is relatively new and is known by several names: adaptronics, adaptive structures, intelligent material systems and structures, smart materials and structures and/or a combination of these words, Smith (2005) and Janocha (2007).
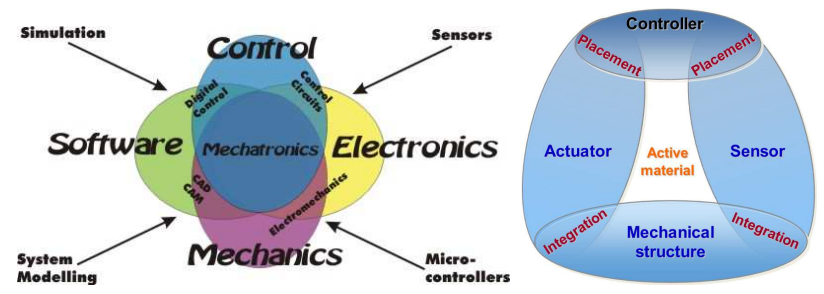

Fig. 1. Mechatronics and smart materials systems design.

The keypoint of such structures is a very high degree of integrability that requires the use of multifunctional and electrically controlled materials, i.e. active materials that can be used indifferently as mechanical structures, actuators or sensors (see Fig. 1). Nevertheless, such materials have a complex and highly non-linear behaviour. This suggests that at least an advanced and in-depth understanding of such behaviours are needed to come up with novel

\footnotetext{
* The contribution of authors has been done within the context of the French National Research Agency sponsored project HAMECMOPSYS number ANR-11-BS03-0002. Further information is available at http://www.hamecmopsys.ens $2 \mathrm{~m} . \mathrm{fr} /$.
}

and efficient devices which have a high-integration degree, and thus, fulfilling mechatronic applications requirements.

This paper is organized as follows. In section 2, we present the main requirements for smart materials based actuators design. The parts constituting such a device are presented to emphasize the necessity to take into account explicitly the interconnection structure between sub-systems in the mechatronic design procedure. But, to model adequately the electromechanical energy conversion process of smart materials, the framework of thermodynamics has to be adopted. Section 3 presents its use in reversible systems whereas the section 4 will extend it in the frame of irreversible thermodynamics. Because smart materials present strong non-linear and hysteretic behaviour, irreversible thermodynamics framework is required for their precise modelling. Finally, some perspectives close the paper to suggest how these modelling procedure can be extended for control purposes. All these works are illustrated using Magnetic Shape Memory Alloys Actuator examples.

\section{INTERCONNECTION AND ENERGY CONVERSION IN SMART MATERIAL SYSTEMS}

Smart material research focus mainly on 6 classes of active materials. From the oldest and the most used to the newest, they are: piezoceramics, magnetostrictives materials, shape memory alloys, electrostrictive polymers, ionic polymers and more recently ferromagnetic shape memory alloys. These materials have different ranges of usability (displacement range, repeatability, output forces, bandwidth, biocompatibility,...) and therefore each have their own niche of application areas. Moreover, it must be 
stressed that none of them, even piezoceramics ${ }^{1}$, behaves in a completely linear manner. Therefore, they have to be modelled using non-linear techniques for high precision applications. Because of space limitations, examples given in this paper deal only with Magnetic Shape Memory Alloys (MSMA).

Even if, smart systems are centered around active materials to increase the degree of integration of the full device, they nevertheless also contain other interconnected components. For example, in the case of an MSMA based actuators as described in Hubert et al. (2012) and displayed in the Fig. 2, the full device is made up of:

- Two active materials (MSMA),

- A transmission structure and a mechanical load,

- A magnetic field generation system (coil/core),

- A power electronic supply system,

- A sensor with its signal processing electronics,

- An electronic control board.

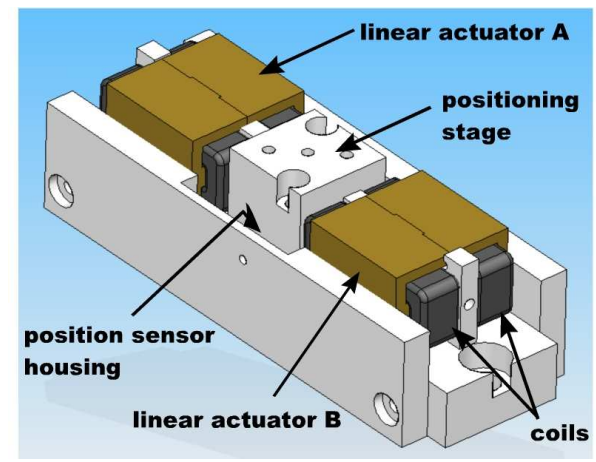

Fig. 2. CAD model of the push-pull actuator using two antagonist MSMA samples in Hubert et al. (2012).

Even if in the near future, integration of such devices improves, smart systems will always be composed of an interconnection of complex subsystems. As a consequence, systems engineering methods (Fiorèse and Meinadier (2012)) and mechatronics design procedure (Isermann (2008)) will continue to be relevant. Indeed, they recommend to explicitly separate the description of interconnection (topological structure of the system) from the behaviour of each subsystems (physical structure and electromechanical energy conversion process) for design and modelling of any complex mechatronics devices.

There are many ways to explicitly describe interconnections between subsystems. The two most commonly used in engineering sciences are linear graph theory with generalized Kirchhoff laws, Shearer et al. (1967) and BondGraph methods, Paynter (1957). A more recent extension of such methods is the Port-Hamiltonian method, Duidam et al. (2009) which combines the energetic point of view of Bond-Graphs with the usefulness of the linear-graph theory. As said previously, it is therefore also essential to separate the topological structure of smart material systems from their physical behaviours. In the frame of MSMA systems, Port-hamiltonian method was recently

\footnotetext{
1 Even if piezoelectric materials are often modelled using linear piezoelectricity equations, most of them as PZT, the most used piezoelectric material for micro-positioning applications, may present hysteresis of around $15 \%$ and creep phenomena.
}

applied in Calchand et al. (2011) to describe the interconnection structure of a MSMA based full device. Considering that such a distinction between interconnection and subsystems behaviour can be done, this paper deals only with physical/constitutive description of smart materials.

As exposed in Crandall et al. (1968) for electromechanical systems and in Preumont (2006) for smart material systems, the relevant framework to model electromechanical energy conversion and actuators behaviour is an energetic framework, such as lagrangian or hamiltonian methods and thermodynamics. This framework is therefore presented in the next two sections for MSMA, starting with reversible processes, we follow with an extension to the thermodynamics of irreversible processes.

\section{ENERGY CONVERSION IN ACTUATORS}

\subsection{The reversible thermodynamics framework}

Each system (or subsystem for a more complex device) can be described by a subset of state variables that completely defines its configuration. Such variables are called extensive variables in the thermodynamics language ${ }^{2}$ and generalized coordinates $q$ in the Lagrangian language. In a static case, a system is completely and uniquely defined by these configuration variables. An energetic state function $\mathcal{U}$ is therefore attached to this configuration, i.e. this state function is a well-defined function of the generalized coordinates. Configuration energy is made up of the potential and internal energy of the system. Nevertheless, at this point, it must be emphasized that actuators are not static but dynamic systems and the set of state variables have to be completed with an other subset of variables, taking into account kinetic effect. Variables of the second type are called generalized momentum $p$. A second energetic state function, the kinetic energy $\mathcal{T}$, is therefore attached to these complementary kinetic state variables. The full state $x$ of the device is completely and uniquely defined by the union of these two subsets $q$ and $p$. The sum of the previous two state functions or the complete state function is called the hamiltonian function of the system:

$$
\mathcal{H}(q, p)=\mathcal{U}+\mathcal{T}
$$

Hamiltonian function is no other than total energy stored in the system.

Energetic state functions are thermodynamic potentials, i.e. they emerge from exact total linear differential forms of state variables. Following the notation of Bamberg and Sternberg (1988), we represents $\omega_{\mathcal{U}}=f(q)[d q]=d \mathcal{U}$, $\omega_{\mathcal{T}}=v(p)[d p]=d \mathcal{T}$ and $d \mathcal{H}=\omega_{\mathcal{U}}+\omega_{\mathcal{T}}=d \mathcal{U}+d \mathcal{T}$, the linear differential forms based on hamiltonian (total energy), configuration and kinetic energy. As exact forms, the energy between two states can be computed as the difference between these two states:

$$
\begin{gathered}
\int_{q_{1}}^{q_{2}} \omega_{\mathcal{U}}=\int_{q_{1}}^{q_{2}} d \mathcal{U}=\mathcal{U}\left(q_{2}\right)-\mathcal{U}\left(q_{1}\right) \\
\int_{p_{1}}^{p_{2}} \omega_{\mathcal{T}}=\int_{p_{1}}^{p_{2}} d \mathcal{T}=\mathcal{T}\left(p_{2}\right)-\mathcal{T}\left(p_{1}\right)
\end{gathered}
$$

\footnotetext{
2 In this context, the thermodynamics framework is more a thermostatic or thermo-quasi-static framework than a "true" thermodynamic one.
} 
As forms, energy stored in the device is:

$$
\begin{aligned}
& \int_{0}^{q} \omega_{\mathcal{U}}=<f, q>=\mathcal{U} \\
& \int_{0}^{p} \omega_{\mathcal{T}}=<v, p>=\mathcal{T}
\end{aligned}
$$

With $<$, $>$, being the duality bracket of these forms. The dual of coordinates $q$, are called generalized forces $f$ whereas the dual of momentum $p$ are called generalized velocity $v$. In this framework, the description of the behaviour of a system is no other than the map linking dual variables. Such maps are called constitutive relations. The constitutive map $f=f(q)$ express the static behavior of the system and the constitutive map $v=v(p)$, express its kinetic behaviour. For reversible processes, these maps are well-defined and invertible. The Fig. 3 depicts the configuration and kinetic behaviour of a thermodynamically reversible system. It must be stressed that for a thermodynamically reversible system, the behaviour/constitutive maps have to be well-defined, i.e. invertible, but they do not need to be linear. A non-linear behaviour can therefore be considered in this framework but a hysteretic behaviour cannot because it is not a single-valued - oneto-one/bijective - map. The key point in such a modelling is that, if we know the total stored energy as a function of coordinates and momenta, the computation of forces and velocities is directly obtained using Hamilton equations:

$$
\begin{aligned}
& v \equiv \dot{q}=\frac{\partial \mathcal{H}}{\partial p} \\
& (-f) \equiv \dot{p}=-\frac{\partial \mathcal{H}}{\partial q}
\end{aligned}
$$

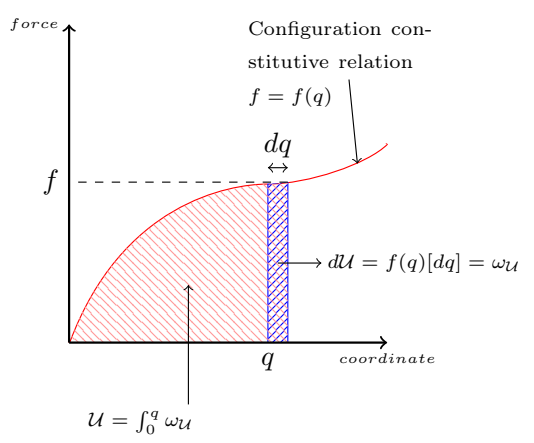

(a)

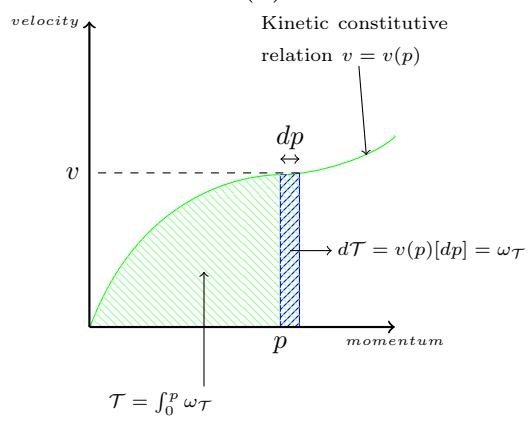

(b)

Fig. 3. Graphical links between dual, state variables, system behaviour and state functions as exact linear differential 1-form: (a) Static/configuration behaviour, (b) kinetic behaviour.
This follows from the fact that the Hamiltonian potential is an exact total differential form of coordinates and momenta and that forces and velocities are duals of theses variables. This principle is quite equivalent to the principle of virtual work extensively used in energy conversion literature because virtual work is no other that the exact differential form $\omega_{\mathcal{U}}=f(q)[d q]=d \mathcal{U}$ and therefore $\frac{\partial \mathcal{H}}{\partial q}=$ $\frac{\partial \mathcal{U}}{\partial q}=f$ by definition. To model an electromechanical energy conversion process, the only thing to do is then to find the adequate Hamiltonian function that describes precisely the physical behaviour of the system, i.e. to find the total energy stored in the device as a function of configuration and kinetic independent variables.

\subsection{Application to MSMA based actuator}

If we consider a simple MSMA based actuator as depicted in Fig. 4 (a) and presented in Gauthier et al. (2008), the different energy functions can be divided into parts as shown in Fig. 4 (b). For reversible system, all dissipation terms have to be neglected, i.e. $Q_{J o u l e}, Q_{\text {hyst }}$ and $Q_{\text {viscous }}$ must not be taken into account. If, in the device, we

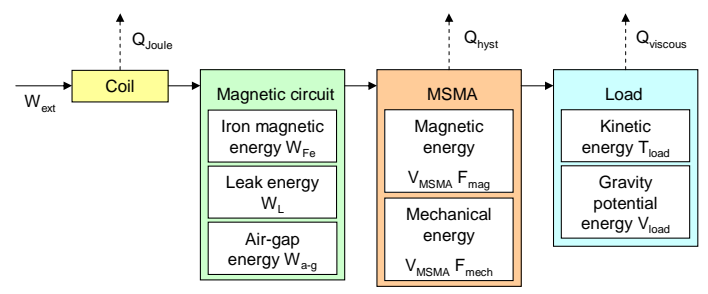

Fig. 4. Example of a simple MSMA based actuator: partition of the different energy contributions, Gauthier et al. (2008).

consider the magnetization energy stored in the iron core, at low magnetic field the behaviour is nearly linear: $B=$ $\mu H$ or $M=\chi H$ with $B, H$, and $M$, being the magnetic induction field, the magnetic excitation field and the magnetization in the core of the magnetic circuit. $\mu$ and $\chi$ are the constant magnetic permeability and the constant magnetic susceptibility of the iron core. Conversely, for higher field level, the behaviour of magnetic materials is no more linear due to saturation of the magnetization $M$ to $M_{s}$. The corresponding energy density, referred as Zeeman energy density, $E_{Z}=\int H d M$, emerges from the differential form $\omega_{Z}=H(M)[d M]=d E_{Z}$ (see Fig. 5).

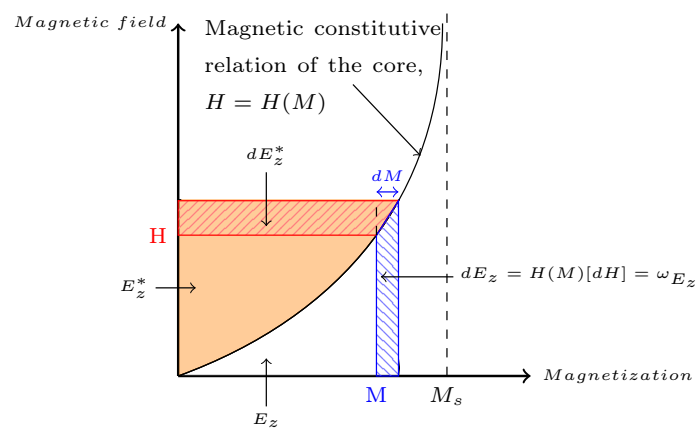

Fig. 5. Zeeman magnetic energy density stored in the core of a magnetic circuit (iron magnetization energy). 
In this example, the generalized coordinate $M$ is considered as the independent variable whereas the generalized force $H$ is the dependent one, i.e. $H$ is a function of $M$ according to the magnetic constitutive law $H=H(M)$. Nevertheless, in practical use, dependent and independent variables do not always correspond to this case. For example, in our actuator, if a reliable current control is implemented, the $H$ field becomes the independent variable in steady-state, because it is directly imposed by current flowing in the coil. In such a case, it is a common practice in reversible thermodynamics to use a Legendre transform to exchange the place of dependent and independent variables. In our case, we will define the magnetization coenergy of the magnetic circuit and in the thermodynamics framework, it corresponds to the definition of a Gibbs free energy potential $\mathcal{G}$. With the definition of $d \mathcal{U}=f(q)[d q]$ and $d \mathcal{U}^{*}=q(f)[d f]$, the magnetization co-energy (a Gibbs potential) is defined as $(-\mathcal{G})=\mathcal{U}^{*}=\int_{0}^{f} d \mathcal{U}^{*}$, making use of the following legendre transform:

$$
\mathcal{U}^{*}=f \cdot q-\mathcal{U}
$$

If we compute, for example, the Zeeman co-energy density of the previous magnetic circuit, it gives $E_{Z}^{*}=M \cdot H-$ $E_{Z}$ as can be seen in the Fig. 5. Let us note however that Legendre transform contains in fact two steps: the first one, computes the complementary area of $\mathcal{U}$ using (5) whereas the second one, expresses the complementary function $\mathcal{U}^{*}$ as a function of the independent variables $f$. This second step necessarily uses the inverse of the constitutive equation $f=f(q)$ to express $q$ as a function of $f$. As noticed previously, this can be done only if the behaviour of the system is reversible and then only if the constitutive map is invertible.

To extend this modelling procedure to the framework of irreversible thermodynamics, we propose a slightly different procedure that gives equivalent results. Instead of using complementary function $\mathcal{U}^{*}$, let us work with an intermediate potential $\tilde{\mathcal{U}}^{*}$ defined by the same Legendre transform but is a function of the dependent $q$ and independent variable $f$ (both are nevertheless considered as independent variables in this intermediate potential):

$$
\tilde{\mathcal{U}}^{*}(f, q)=f \cdot q-\mathcal{U}(q)
$$

When subjected to the following behaviour constraint $\frac{\partial \tilde{\mathcal{U}}^{*}(f, q)}{\partial q}=0$, this intermediate potential carry in fact the same information as $\mathcal{U}^{*}(f)$ because:

$$
\begin{aligned}
& \frac{\partial \tilde{\mathcal{U}}^{*}(f, q)}{\partial q}=0 \\
& \Rightarrow \frac{\partial(f \cdot q-\mathcal{U}(q))}{\partial q}=0 \\
& \Rightarrow f-\frac{\partial \mathcal{U}(q)}{\partial q}=0 \\
& \Rightarrow f=\frac{\partial \mathcal{U}(q)}{\partial q}
\end{aligned}
$$

The definition of $f$ through the exact differential form $\omega_{\mathcal{U}}$ of the variable $q$ is therefore a consequence of this constitutive constraint. Moreover, as noticed by Smith (2005), because:

$$
\frac{\partial \tilde{\mathcal{U}}^{*}(f, q)}{\partial f}=\frac{\partial(f \cdot q-\mathcal{U}(q))}{\partial f}=q,
$$

the thermodynamic constitutive relation $\frac{\partial \tilde{\mathcal{U}}^{*}(f, q)}{\partial f}=q$ can be interpreted as the necessary condition to minimize $\tilde{\mathcal{U}}^{*}(f, q)$ under the condition that $\mathcal{U}$ is a function of $q$ (see Fig. 6). This procedure presents the great advantage of using the complementary potential without the necessity to inverse the constitutive relation.

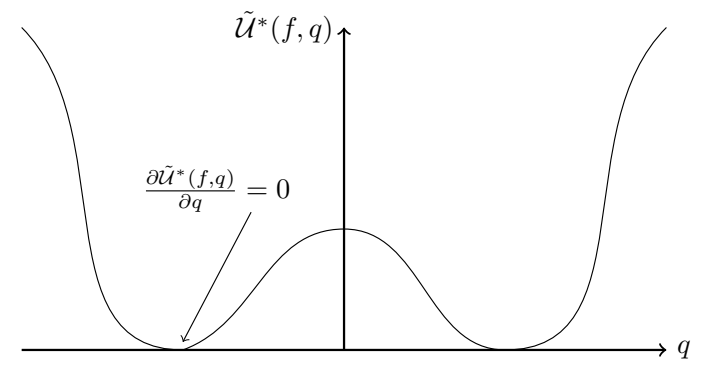

Fig. 6. Under the condition that $\mathcal{U}$ is a function of $q$, the thermodynamic constitutive relation $\frac{\partial \tilde{\mathcal{U}}^{*}(f, q)}{\partial f}=q$ can be interpreted as the necessary condition to minimize $\tilde{\mathcal{U}}^{*}(f, q)$ according to $q$, i.e. $\frac{\partial \tilde{\mathcal{U}}^{*}(f, q)}{\partial q}=0$.

With this slight modification in the modelling procedure, it is now possible to add any non-independent variable $y$ to describe a complementary thermodynamic potential $\tilde{\mathcal{U}}^{*}$ and at the same time, add a constitutive constraint $\frac{\partial \tilde{\mathcal{U}}^{*}(f, q, y)}{\partial y}=0$ that ensures system behaviour is respected. The constitutive relations is, in this case, computable using (8). This alternative way of writing the behaviour and constitutive laws, allows to extend this modelling procedure to the frame of irreversible behaviour. Indeed, the inversion of the behaviour/constitutive map is no more necessary: the framework can be used even for noninvertible and hysteretic behaviour.

\section{IRREVERSIBLE THERMODYNAMICS AND SMART MATERIAL BEHAVIOUR}

As explained in the introduction of the paper, all smart materials present non-linear and hysteretical behaviour. In the case of MSMA, the Fig. 7 reports experimental measurements of constitutive behaviour for NiMnGa sample (the strain $\varepsilon$ is the generalized coordinate and the stress $\sigma$, its dual generalized force). As can be seen, the differential form $\omega_{\mathcal{W}}(\varepsilon)[d \varepsilon]$ associated with the virtual work $d \mathcal{W}$ supplied to the sample is not an exact total form ${ }^{3}$. Integration of the work between two states depends therefore on the integration path and gives rise to energy dissipation $\mathcal{D}$ and increase in irreversible entropy.

\subsection{From reversible to irreversible behaviour modelling}

Following the seminal paper of Coleman and Gurtin (1967), the idea of irreversible thermodynamics with internal variables is to find additional state variables that can explain the irreversible behaviour mechanisms and compute the dissipation functions associated with any irreversible processes. Such kind of dissipation functions $\mathcal{D}$ are

\footnotetext{
3 The sign $d$ is sometimes used in irreversible thermodynamics to specify that the corresponding form is not an exact form.
} 

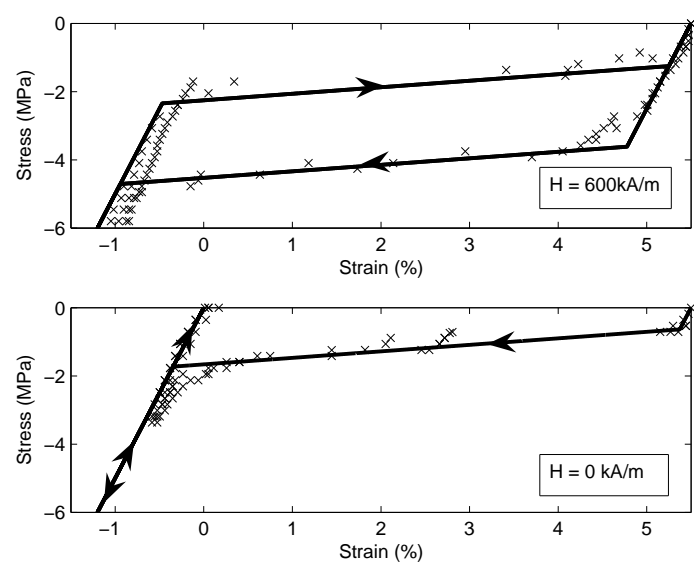

Fig. 7. Mechanical stress versus strain curves with and without magnetic field for a cyclic charging (simulation: solid line, experimental results: cross points), Gauthier et al. (2008).

similar to Rayleigh dissipation functions. Most of the time, such additional internal state variables are inspired by microscopic considerations and homogenization techniques (see Maugin (1999)).

These additional state variables $y$ could be added to any thermodynamics potential $\Phi$ as explained in the previous sections. If such additional variables are not independent, they have to be associated with behavioural constraints $\frac{\partial \tilde{\Phi}(f, q, y)}{\partial y}=0$ to ensure system behaviour. In such a case, they can only be used to explain more clearly the internal complex behaviour but not to quantify the effects. If these additional variables are independent variables, they are associated with behaviour constraints $\frac{\partial \tilde{\Phi}(f, q, y)}{\partial y} \neq 0$. Such internal independent variables are therefore usable to explain and calculate the source of irreversibility during any variation. Associated constitutive equations $\frac{\partial \tilde{\Phi}(f, q, y)}{\partial y}=f_{y}$ defines a corresponding dual generalized internal force $f_{y}$. In this new framework, the Clausius-Duhem inequality is then an efficient way to quantify the dissipation potential as:

$$
d \mathcal{D}=\omega_{\mathcal{D}}(y)[d y]=<f_{y}, d y>\geq 0
$$

The dissipation due to irreversible processes is directly linked to the area (necessary positive, according to the second law of thermodynamics) inside hysteretic loop in behaviour/constitutive law (see Fig. 7). When reversible behaviours were considered in the previous sections, dissipation were taken to be nul. Experimental/phenomenological measurements are nevertheless required to find adequate constitutive dissipation law using these additional state internal variables.

\subsection{Application to MSMA based actuator}

Good descriptions of internal variables used to model irreversible behaviour in MSMA smart materials are presented in Gauthier et al. (2007) and Sarawate and Dapino (2010). Three internal variables are required to model a simplified two-variants microstructure of an MSMA sample: two internal variables $\alpha$ and $\theta$ are reversible (dependent variables) whereas the third internal variable $\xi$ is related to the irreversible behavior (independent variable). The two dependent internal state variables $\alpha$ and $\theta$ describes more clearly the complex magnetic behaviour of MSMA material. $\alpha$ helps to explain the Weiss domain-like microstructure of the material and it is used to calculate the magneto-static energy of the MSMA sample. $\theta$ models the rotation of the magnetization fields inside Weiss domains and is used in the quantification of the anisotropic crystallographic energy of the sample. These two variables helps to take into account magnetic saturation phenomena in MSMA easily. The third independent internal variable $\xi$ is the volume fraction of one variant with respect to the other. The knowledge of $\xi$ explains the reorientation of martensite variants inside MSMA sample and permits to compute the rearrangement strain of the sample. As an independent variable, a constitutive equation of its behaviour has to be defined/measured experimentaly. As can be seen in Fig. 8, these three internal variables are inspired by the microstructure of MSMA sample (NiMnGa material) and are used to clarify the electromechanical energy conversion inside this smart material.

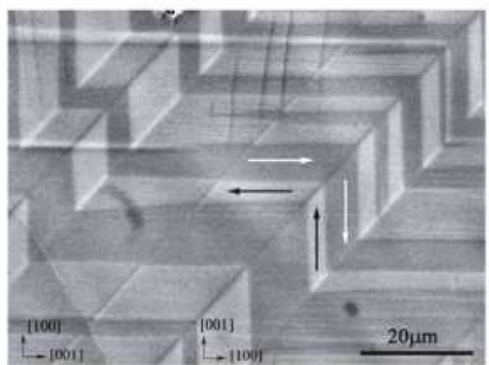

(a)

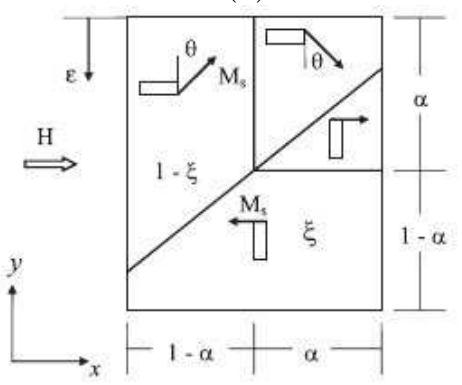

(b)

Fig. 8. Internal variables and cristallographic microstructure of NiMnGa MSMA: (a) Image of twin-variant NiMnGa microstructure by scanning electron microscope, (b) Simplified two-variant microstructure of NiMnGa (Representative Volume Element). Sarawate and Dapino (2010).

The constitutive equation of the independent internal variable $\xi$ describes the irreversible behaviour of MSMA and is used to calculate the dissipation function $\mathcal{D}$. In Gauthier et al. (2008), a simple hysteresis loop was chosen, where threshold and rate were identified experimentally (see Fig. 9).

If the need arises, more complex constitutive behaviours can be considered according to experimental measurements. Nevertheless, each choice of constitutive equations has to be associated with an experimental identification procedure. Therefore, complex constitutive equations require complex identification procedures. We are currently 


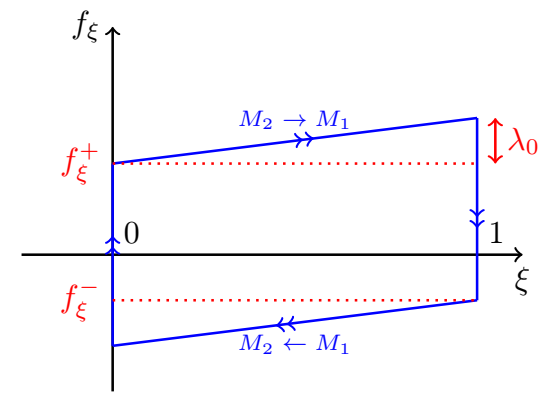

Fig. 9. Exemple of constitutive law for irreversible internal state variable: coordinate $\xi$ versus dual internal force $f_{\xi}$, Gauthier et al. (2008).

working on the definition of constitutive laws able to take into account hysteresis behaviour with internal loops. Calchand et al. (2012) presents a first attempt in this direction, using Preisach-like constitutive models associated to the $\xi$ internal state variables. We hope that this will improve the irreversible behaviour description of MSMA smart material systems in the near future. This thermodynamically consistent modelling method could then be used for control purposes by applying energy-based control methods.

\section{CONCLUSION}

This paper presents a new class of device termed smart systems. They are based on active materials and permit the design of high performance and highly integrated mechatronic devices. Nevertheless, one of the main difficulty in their design is to take into account the complex nonlinear and irreversible behaviour of active materials. After a review of reversible thermodynamics for energy conversion, it was shown that irreversible thermodynamics with internal variables can be used to overcome such difficulties. Examples using Magnetic Shape Memory Alloys actuators illustrate this modelling procedure. Actual results are encouraging but improvements are still needed to take into account internal loops for hysteretic behaviours and dynamics effects. Nevertheless, efficient extensions in the frame of mathematical systems theory can be envisaged for control purposes.

\section{ACKNOWLEDGEMENTS}

Authors would like to thank Prof. C. Lexcellent, Femto-ST Institute, Besançon, France, for collaborations concerning the modeling of SMA and MSMA, and Prof. B. Mashke, LAGEP laboratory, University of Lyon, France, for discussions concerning interconnexion structures and PortHamiltonian Methods.

\section{REFERENCES}

Bamberg, P. and Sternberg, S. (1988). A course of mathematics for students of physics. Cambridge University Press.

Calchand, N., Hubert, A., and Le Gorrec, Y. (2012). Port hamiltonian modeling of msma based actuator: toward a thermodynamically consistent formulation. In IFAC Lagrangian and hamiltonian method for non linear control Conference (LHMNLC 2012). , Italy.
Calchand, N., Hubert, A., Le Gorrec, Y., and Mashke, B. (2011). From canonical hamiltonian to port-hamiltonian modeling. Application to magnetic shape memory alloys actuators. In ASME 2011 Dynamic Systems and Control Conference (DSCC'2011). Airlington, Virginia, USA.

Coleman, B.D. and Gurtin, M.E. (1967). Thermodynamics with internal state variables. J. Chem. Phys., 47, 597613.

Crandall, S.H., Karnopp, D.C., Krutz, J.E.F., and Pridmore-Brown, D.C. (1968). Dynamics of Mechanical and Electromechanical Systems. McGraw-Hill Inc., New York.

Duidam, V., Macchelli, A., Stramigioli, S., and Bruyninckx, H. (eds.) (2009). Modeling and control of complex physical systems. The port-hamiltonian approach. Springer Verlag.

Fiorèse, S. and Meinadier, J.P. (eds.) (2012). Découvrir et comprendre l'ingénierie système. Cépaduès Éditions.

Gauthier, J.Y., Hubert, A., Abadie, J., Chaillet, N., and Lexcellent, C. (2008). Nonlinear hamiltonian modelling of magnetic shape memory alloy based actuators. Sensors \& Actuators A, 141, 536-547.

Gauthier, J.Y., Lexcellent, C., Hubert, A., Abadie, J., and Chaillet, N. (2007). Modeling rearrangement process of martensite platelets in a magnetic shape memory alloy $\mathrm{Ni}_{2} \mathrm{MnGa}$ single crystal under magnetic field and (or) stress action. Journal of Intelligent Material Systems and Structures, 18(3), 289-299.

Hubert, A., Calchand, N., Gorrec, Y.L., and Gauthier, J.Y. (2012). Magnetic shape memory alloys as smart materials for micro-positioning devices. Advanced electromagnetics, $1(2), 75-84$.

Isermann, R. (2008). Mechatronics systems - innovative products with embedded control. In Proceedings of the IFAC Mechatronics conference.

Janocha, H. (2007). Adaptronics and Smart Structures - Basics, Materials, Design and Applications, 2nd ed. Springer-Verlag.

Maugin, G. (1999). The thermomechanics of nonlinear irreversible behaviours. An Introduction. World scientific.

Paynter, H.M. (1957). Analysis and Design of Engineering Systems. MIT Press, Cambridge, Mass.

Preumont, A. (2006). Mechatronics : Dynamics of Electromechanical and Piezoelectric Systems. Springer Verlag.

Sarawate, N.N. and Dapino, M.J. (2010). Magnetomechanical characterization and unified energy model for the quasistatic behaviour of ferromagnetic shape memory ni-mn-ga. Smart Materials and Structures, 19.

Shearer, J.L., Murphy, A.T., and Richardson, H.H. (1967). Introduction to system dynamics. Addisson-Wesley.

Smith, R.C. (2005). Smart Material Systems. Model Development. SIAM Frontiers in applied mathematics. 